Konstanze Söllner

\title{
Der Deutsche Bibliothekartag. Netzwerk und Inkubator für den Wandel
}

Der Deutsche Bibliothekartag als größte bibliothekarische Fortbildungsveranstaltung in Europa zieht seit seinem 100. Jubiläum im Jahr 2011 in Berlin regelmäßig rund 4000 Teilnehmerinnen und Teilnehmer an. Aus Branchensicht stellt der Bibliothekartag eine Großveranstaltung dar und gehört mit einer Teilnehmerzahl von mehr als 1000 Gästen und einer Veranstaltungslänge von mehr als zwei Tagen bereits zu den absoluten Ausnahmeereignissen, wie die vergleichende Übersicht in Tab. 1 veranschaulicht.

Tab. 1: Vergleich der Teilnehmerzahlen von Großveranstaltungen und so genannten Mega-Kongressen 2016/2017.

\begin{tabular}{lr}
\hline Soziologiekongress & 2200 \\
\hline Juristentag & 2200 \\
\hline Deutscher Logistik-Kongress & 3000 \\
\hline Historikertag & 3800 \\
\hline Bibliothekartag & 3800 \\
\hline Neurologiekongress & 6000 \\
\hline Re:publica & 9000 \\
\hline Chaos Communication Congress & über 10000 \\
\hline
\end{tabular}

Wie die meisten großen Kongresse ist der Bibliothekartag wissenschaftlich orientiert, entwickelt sich aber immer mehr zu einer Fortbildungsveranstaltung mit didaktischen Elementen. Zugleich dient er der Abwicklung von Verbandsregularien (Mitgliederversammlungen). Als zentrale Fortbildungsveranstaltung erfüllt er aber auch eine Doppelfunktion, indem er nicht nur der individuellen Weiterbildung und dem persönlichen Networking dient, sondern ebenso eine Plattform für die Lobbyarbeit bildet. Weitgehend unbemerkt von den Kongressteilnehmern dient der Bibliothekartag immer wieder als Vehikel für die Kommunikation zentraler Themen des deutschen Bibliothekswesens, seien es ein novelliertes Urheberrecht, das Recht auf den Verleih von E-Books oder veränderte Arbeitsbedingungen in Bibliotheken. Dennoch verhält es sich beim Biblio-

1 Vgl. dazu: Hundhausen, Felicitas, Daniela Lülfing u. Wilfried Sühl-Strohmenger (Hrsg.): 100. Deutscher Bibliothekartag. Festschrift. Hildesheim, Zürich, New York: Olms 2011.

ə Open Access. (C) 2018 Konstanze Söllner, publiziert von De Gruyter. (G) BY-NC-ND Dieses Werk ist lizenziert unter der Creative Commons Attribution-NonCommercial-NoDerivatives 4.0 Lizenz. 
thekartag nicht so wie bei manchen Hotspots der Kongressszene, wo der Inhalt gegenüber Networking, Kommunikation und Lobbyarbeit weitgehend nebensächlich wird. Das zeigt auch die ständig steigende Zahl der Einreichungen (Nürnberg 2015: 344, Frankfurt am Main 2017: 392, Berlin 2018: 460).

\section{Kongressbesuch vs. Internetkommunikation}

Im Zeitalter ubiquitärer Informationsangebote in Form von Blogs, Mikrobloggingdiensten, sozialen Netzwerken bis hin zu Onlinezeitschriften stellt sich schnell die Frage nach dem Sinn von Kongressbesuchen. Wie gelingt es dem Bibliothekartag, die Angehörigen bibliothekarischer und verwandter Berufe zu mobilisieren, sodass sie sich nicht auf das ortsunabhängige Informations- und Kommunikationsangebot des Internets verlassen, sondern Kosten und Mühen auf sich nehmen, um sich live in einer meist weit entfernten Stadt zu treffen?

Der Bibliothekartag setzt sich traditionell aus Konferenz und begleitender Messe zusammen, ein Angebot, das nicht ohne Weiteres virtualisiert werden kann. Damit ist er der Ort für die laufende „Marktbeobachtung“, sowohl was die Entwicklung der Branche als auch das Produkt- und Dienstleistungsangebot von Firmen angeht. Auch kann man davon ausgehen, dass Kongressbesucher insgesamt ein höheres Informationsinteresse haben als Personen, die nur raumungebundene Informationsangebote nutzen. Die Teilnehmerinnen und Teilnehmer tragen damit selbst zur Attraktivität der Veranstaltung nicht wenig bei, weil sie absichern, dass eine gut informierte Community vor Ort anzutreffen ist und dass eine hohe Informationsdichte innerhalb der Kongressdauer erreicht wird. Der Bibliothekartag bezieht folglich seine Attraktivität ganz wesentlich daraus, dass man dort Kolleginnen und Kollegen mit profundem Fachwissen antrifft. Tagungspausen und Networking-Events spielen bei diesem Vorgang keine geringe Rolle. Der Bibliothekartag kann als Kongress folglich nur gelingen, wenn dort konkrete und drängende Themen und Fragestellungen von Referentinnen und Referenten behandelt werden, die in ihrem Arbeitsbereich fachlich führend sind. Als durchmoderierte Infotainment-Show hätte der Bibliothekartag seinen Zweck verfehlt.

Kongresse haben eine weitere wichtige Funktion, indem sie auf eine Community ebenso aufmerksam machen wie auf Branchenveränderungen und wichtige Einflussgrößen, wie etwa das rechtliche oder tarifliche Umfeld. Gleichzeitig tragen sie dazu bei, die Fachcommunity zu konstituieren. Geht man davon aus, dass es in Deutschland ca. 35000 bis 40000 Angehörige bibliotheka- 
rischer Berufe gibt, ${ }^{2}$ so erreicht der Bibliothekartag regelmäßig ein Zehntel der gesamten Berufsgruppe - ein Wert, der außergewöhnlich hoch ist und die enorme Durchdringungskraft der Veranstaltung im Hinblick auf die Zielgruppe zeigt. Selbst diejenigen, die den Bibliothekartag nicht besuchen, informieren sich über das Kongressgeschehen in Quasi-Echtzeit durch das Nachlesen veröffentlichter Präsentationsfolien und Blogarchive oder durch das Verfolgen von Hashtags.

\section{Die Teilnehmerinnen und Teilnehmer}

Der Deutsche Bibliothekartag wurde im Jahr 1900 als zentrale Fortbildungsveranstaltung des VDB - Verein Deutscher Bibliothekarinnen und Bibliothekare begründet. Er findet jährlich an verschiedenen Orten statt, aller drei Jahre turnusmäßig als Bibliothekskongress der in Bibliothek \& Information Deutschland (BID) zusammengeschlossenen bibliothekarischen Verbände. Von 1951 bis 2000 wurde der Bibliothekartag vom VDB gemeinsam mit dem Verein der Diplom-Bibliothekare an wissenschaftlichen Bibliotheken (VdDB) veranstaltet, ${ }^{3}$ seit 2001 gemeinsam mit dessen Nachfolgeorganisation Berufsverband Information Bibliothek (BIB). ${ }^{4}$ War der Bibliothekartag zunächst eine Veranstaltung des wissenschaftlichen Bibliothekswesens, so hat er sich in den letzten Jahrzehnten zu einer spartenübergreifenden Tagung und multiprofessionellen Veranstaltung mit großer Praxisnähe entwickelt. Dennoch wird der Bibliothekartag weit häufiger von Beschäftigten wissenschaftlicher Bibliotheken besucht als von Beschäftigten öffentlicher Bibliotheken, wie Abb. 1 veranschaulicht. In den Jahren 2015 bis 2017 kamen beim Bibliothekartag ebenso wie beim Bibliothekskongress $65 \%$ aller Fachbesucherinnen und Fachbesucher aus wissenschaftlichen, jedoch nur $15 \%$ bis $18 \%$ aus öffentlichen Bibliotheken. Es ist davon auszugehen, dass die Besucherinnen und Besucher aus öffentlichen Bibliotheken vor allem aus der Leitungsebene stammen, während eine Kongressteilnahme in den wis-

2 Berufe im Spiegel der Statistik: Berufsordnung 823 Bibliothekare/innen, Archivare/innen, Museumsfachleute. http://bisds.infosys.iab.de/bisds/result?region=19\&beruf=BO823\&qualifikation=2. Alle aufgeführten Internetressourcen wurden zuletzt am 9. Dezember 2017 aufgerufen.

3 Vgl. dazu auch Reisser, Michael u. Susanne Riedel: „Nur was sich ändert, bleibt!“ - Einige Gedanken zum Bibliothekartag gestern, heute und vor allem morgen. In: 100. Deutscher Bibliothekartag (wie Anm. 1). S. 39-48.

4 Von 2008 bis 2016 bestand eine Kooperation mit dem Deutschen Bibliotheksverband, der 2018 erstmals einen eigenen bundespolitischen Kongress veranstaltet. 
senschaftlichen Bibliotheken zum Fortbildungsalltag einer größeren Gruppe von Mitarbeiterinnen und Mitarbeitern gehört.

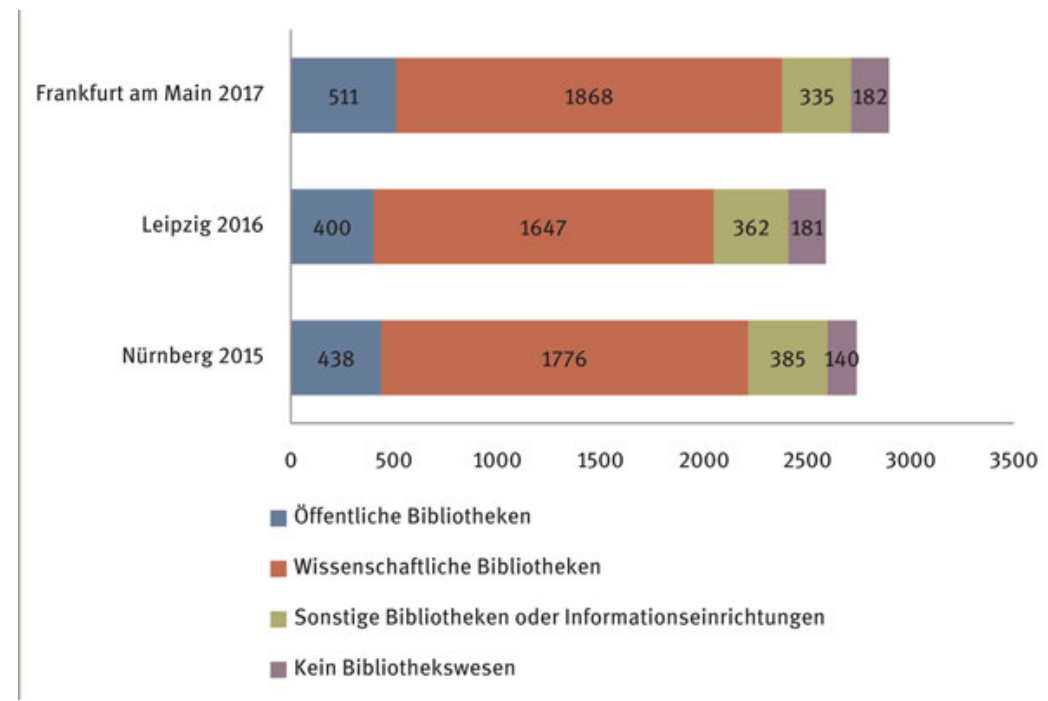

Abb. 1: Fachbesucherinnen und Fachbesucher nach Bibliothekssparten.

Die regionale Verteilung der Teilnehmerinnen und Teilnehmer entspricht der Größe der Berufsgruppe in den Ländern, wie in Abb. 2 dargestellt. So ist es nicht überraschend, dass das Land Berlin (13\%) gleichauf mit Bayern (13\%) auf dem zweiten Platz hinter Nordrhein-Westfalen (14\%) rangiert, wiederum gefolgt von Baden-Württemberg (12\%) und Hessen (11\%). 

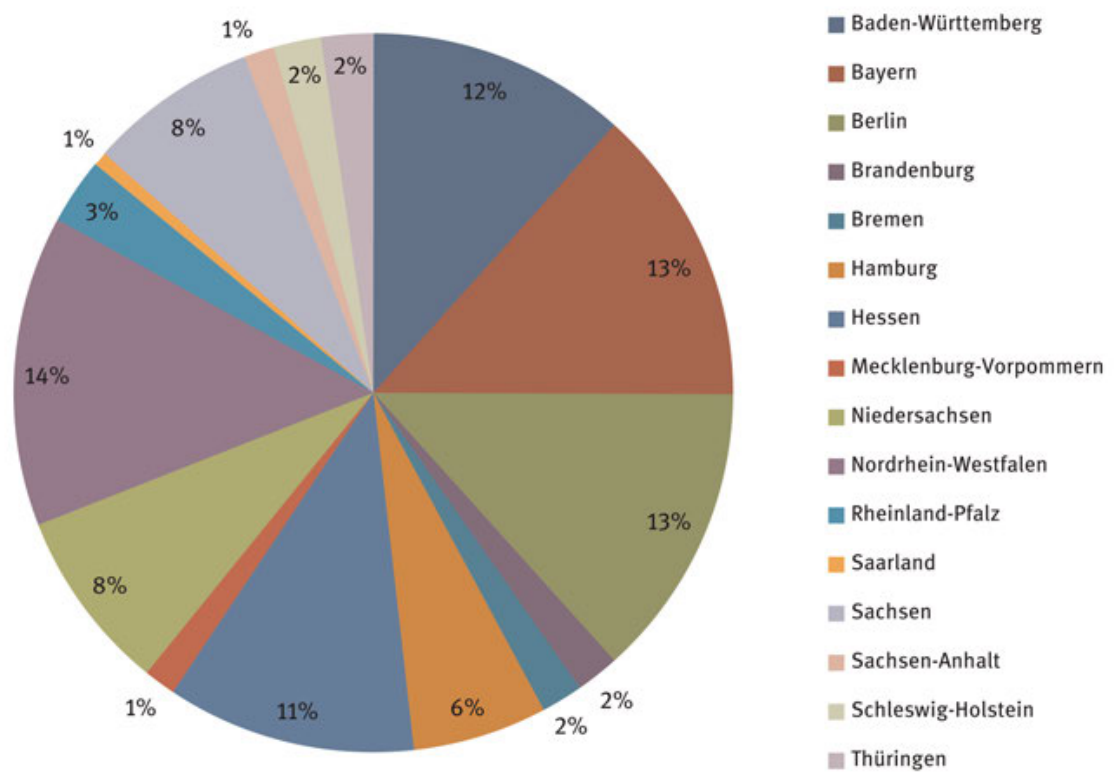

Abb. 2: Zugehörigkeit der Teilnehmerinnen und Teilnehmer nach Bundesländern.

Allerdings verschieben sich die regionalen Anteile erheblich mit dem Ortswechsel des Bibliothekartags. Findet der Bibliothekartag im eigenen Bundesland statt, erhöht sich der Anteil der Teilnehmerinnen und Teilnehmer aus diesem Land relativ gesehen wenigstens auf das Doppelte. Wenn der Bibliothekartag im unmittelbar benachbarten Bundesland stattfindet, führt auch dies, wie in Abb. 3 veranschaulicht, zu einer vermehrten Teilnahme, so etwa 2015 und 2017 durch baden-württembergische Fachbesucherinnen und Fachbesucher in Nürnberg und Frankfurt am Main. Dies zeigt, dass die regionale Mobilität vieler Bibliothekarinnen und Bibliothekare - was die Fortbildungsteilnahme angeht nicht sehr ausgeprägt ist und dass man sich bevorzugt im eigenen Bundesland oder in den benachbarten Bundesländern fortbildet. Nicht nur deshalb muss der Bibliothekartag an wechselnden Orten stattfinden, will er die Community auch unabhängig von der regionalen Verteilung der Bibliotheken erreichen. 


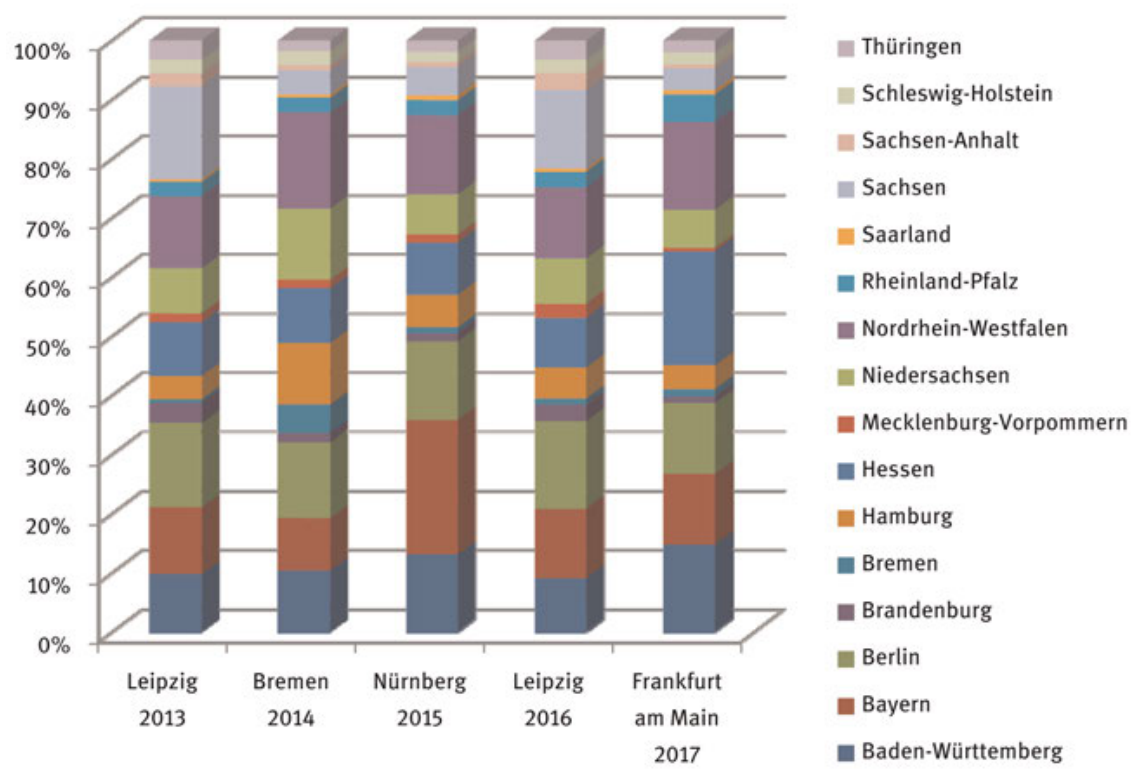

Abb. 3: Teilnahme in Abhängigkeit von der regionalen Zugehörigkeit.

\section{Wie groß darf die inhaltliche Breite sein, wo bleibt die Synthese?}

Der hohe Grad an Durchdringung der bibliothekarischen Community, über die unterschiedlichen Qualifikationen und Berufe hinweg, führt naturgemäß zu einem vielfältigen inhaltlichen Angebot. Die günstige Kostenstruktur beim Bibliothekartag ermöglicht zusätzlich eine breite Teilnehmerschaft. Der Call for Papers, der nahezu alle Themen des Bibliothekswesens aufgreift, sorgt immer wieder für eine große Zahl an Einreichungen, auch aus Bereichen, die die Veranstalter zunächst weniger „auf dem Schirm“ hatten, und damit zwangsläufig für ein sehr breit gefächertes Programm. Für die Veranstalter stellt der offene Zugang für alle Einreichenden ein wichtiges Handlungsmotiv dar, und nicht selten wurden fertige Sessions der eigenen Kommissionen $\mathrm{zu}$ deren nicht geringer Enttäuschung neu aufgeteilt, um inhaltliche Bezüge zu anderen Einreichungen herzustellen. Die schiere Menge an Beiträgen führt zu sich wiederholenden Klagen über zeitliche Überschneidungen von Themenblöcken, sodass es nicht verwundert, dass der Ruf nach einer stärkeren Fokussierung immer 
wieder laut wird. Dem versuchten die Verbände in den letzten Jahren durch in Eigenverantwortung kuratierte „Invited Sessions“ zu entsprechen. Aber nicht nur eine stärkere Steuerung und Kuratierung der Programminhalte wird notwendig sein, um deutlichere Linien im Kongressprogramm zu ziehen, auch die Herbeiführung einer inhaltlichen Synthese gehört zu den wichtigsten Aufgaben für die Kongressorganisation der kommenden Jahre. Dabei geht es nicht nur um die anschließende Publikation von Vorträgen in einem Tagungsband, sondern um ein integrierendes Vorgehen bereits bei der Kongressvorbereitung. Eine strategisch angelegte Fokussierung auf Themen oder Gruppen führt zwangsläufig zu einer Ausgrenzung bestimmter Bereiche. Dies zeigte etwa die Entstehung des virtuellen Angebots „Bibcast“ im Umfeld des Leipziger Bibliothekskongresses 2016. Bei diesem Kongress wurde gezielt das Thema „Bibliothekräume - real und digital" fokussiert. Aus den abgelehnten Vorträgen entstand in einem Bottom-up-Prozess eine Sammlung von Webcasts mit begleitenden Etherpads zur Vorbereitung und Diskussion nach dem Vorbild der IT-Konferenz „Reject. JS“. Die Verluste an thematischer Vielfalt können ebenso dazu führen, dass man mit Themen, die im eigenen Berufsalltag nicht vorkommen, aber womöglich dennoch für diesen relevant sind, selbst auf dem Bibliothekartag nicht mehr in Kontakt kommt. Allerdings wäre bereits jetzt ein enormer Spagat zwischen Themen wie Forschungsdatenmanagement und Bibliothekspädagogik, Digital Humanities und Leseförderung nötig, will man einen Gesamtüberblick erhalten - ein Spagat, der in der Realität nicht stattfindet und auch gar nicht stattfinden kann.

Besondere Chancen einer derartig großen Veranstaltung bestehen im Lancieren von Leitthemen, die Brisanz haben und viele potenzielle Teilnehmerinnen und Teilnehmer betreffen, die aber zugleich die praktische Wissensvermittlung ermöglichen. Ein Alternieren von Leitthemen wäre möglich und denkbar, durchaus auf Kosten der Größe der Veranstaltung, aber dafür mit klarer Schwerpunktsetzung. Dieses hätte allerdings zur Voraussetzung, dass das Kongressprogramm noch stärker vorstrukturiert und langfristiger vorbereitet wird, um den Themenschwerpunkten tatsächlich gerecht zu werden und das Programm nicht von mehr oder minder zufälligen Einreichungen bestimmen zu lassen. Damit verbunden wäre der Verzicht auf thematisch weniger einschlägige Einreichungen, so relevant und aktuell diese auch sein mögen. Damit würde der Bibliothekartag noch stärker als bisher den Charakter einer vorbereiteten Fortbildungsveranstaltung als den eines quasi wissenschaftlichen Kongresses annehmen, auf dem die neuesten Themen in der Breite vorgestellt werden, wie sie eben eingehen und aus Sicht der Gutachterinnen und Gutachter vortragsreif und relevant sind. Der Bibliothekartag wäre dann weniger Quelle zur Aufdeckung von Neuerungen und Trends und somit des eigentlichen Fortbildungsbe- 
darfes als vielmehr eine in didaktischer Hinsicht gut vorbereitete Themenveranstaltung mit einem gezielt gewählten Nebeneinander von verschiedenen Veranstaltungstypen, die intensiver als bisher die Teilnehmerinnen und Teilnehmer aktivieren und sie mit anwendungsbereitem Wissen versorgen. Es wäre der Schritt weg von der großen Plattform für mal mehr, mal weniger gelingende Sessions, Podiumsdiskussionen, kleine Sitzungen oder Kommissionsveranstaltungen hin zu einem Kongressprogramm mit besser ausgeprägter Partizipation, mehr didaktischen Elementen, aber zugleich mit stärker vorhersehbaren Inhalten und Ergebnissen. Dies könnte durchaus im Interesse der entsendenden Einrichtungen sein, wenn die Qualität stimmt, bedeutet aber auch, dass der Bibliothekartag einen Teil seines Charakters als Kongress und offenes Diskussionsforum verliert, zugunsten eines Programms von durchstrukturierten Lernund Arbeitseinheiten.

\section{Rahmenbedingungen - der Bibliothekartag als Ort}

Gerade Wachstumsbranchen - und dazu gehört der Bildungs- und Hochschulbereich - haben einen hohen Bedarf an Wissenstransfer. Beim Bibliothekartag findet der Wissenstransfer sowohl im Rahmen der Fortbildungsveranstaltung als auch bei der Firmenausstellung statt. Der Nachteil von Tagungen, Konferenzen und Messen besteht allerdings darin, dass diese nicht disponibel, sondern orts- und zeitabhängig sind. Beim Bibliothekartag wird wie bei anderen Veranstaltungen die Attraktivität danach beurteilt, wie viele Teilnehmende er angezogen hat. Die Mobilisierung potenzieller Besucherinnen und Besucher steht im Zeichen ubiquitärer Informationsangebote und stellt die eigentliche Herausforderung der nächsten Jahre dar. Die infrastrukturelle Passung (Anreise, Hotelverfügbarkeit und -kosten) spielt deshalb bei der Mobilisierung der Fachbesucherinnen und Fachbesucher keine geringe Rolle, weil sie die Hemmschwelle zur Partizipation durch Senkung des individuellen Aufwands herabsetzt.

Die Suche nach der passenden Destination bezieht diese Überlegungen mit ein und beginnt immer schon einige Jahre vor dem eigentlichen Bibliothekartag. Die Festlegung des Kongresszentrums findet drei bis vier Jahre vor dem Kongress statt. Seit dem 95. Bibliothekartag 2006 in Dresden finden die Bibliothekartage nur noch in professionellen Kongresszentren statt. Die wichtigsten Gründe dafür sind die wachsende Zahl der Teilnehmerinnen und Teilnehmer 
und der Bedarf an Tagungsräumen sowie professionellen Ausstellungs- und Standflächen in großen Messehallen. Kongresszentrum plus marktgerechte Hotellerie mit möglichst zentraler Lage und kurzen Wegen stellen die veranstaltungswirksame Infrastruktur vieler Messe und Kongresse, nicht zuletzt auch der Großveranstaltung Deutscher Bibliothekartag. Dabei haben bedeutende Kongresszentren häufig hohe Preisvorstellungen - allerdings nicht zwingend, wie die Erfahrung der Vergangenheit zeigt. Die eigentliche Vorbereitung des Bibliothekartags dauert schließlich ein gutes Jahr, wobei der lokalen Verankerung eine wichtige Rolle zukommt. Der Wechsel zwischen den Regionen ist nicht nur wichtig für die breite regionale Erreichbarkeit der Community, er ermöglicht ebenso die Unterstützung durch die Häuser vor Ort, damit die Belastung für einzelne Bibliotheken nicht zu hoch wird. Ohne die Unterstützung durch die örtlichen Bibliotheken insbesondere mit Räumen für eine Vielzahl an Gremiensitzungen (charakteristisch für den Bibliothekartag ist ein hoher Bedarf an mittelgroßen und kleineren Vortragssälen und Tagungsräumen) und mit Personal in Gestalt freiwilliger Helfer, die zum Ausgleich für ihren Einsatz eine kostenfreie Registrierung über die gesamte Kongressdauer erhalten, ist der Bibliothekartag wohl nicht durchführbar, soll er für die Teilnehmerinnen und Teilnehmer erschwinglich bleiben. Aber auch die inhaltliche Mitarbeit durch die Bibliotheken vor Ort spielt eine zentrale Rolle - angefangen bei der Eröffnungsveranstaltung und der Ansprache der örtlichen Politik über Führungen und Veranstaltungen in den Bibliotheken bis hin zur Beteiligung im Programmkomitee des Bibliothekartags oder am Programm des Bibliothekartags selbst. ${ }^{5}$

\section{Wirkung und Nutzen des Bibliothekartags}

Angesichts des hohen organisatorischen Aufwands stellt sich die Frage nach Wirkung und messbarem Nutzen der Bibliothekartage. Für den durchschnittlichen Besucher einer der üblichen Messen spielt der Kongressbesuch eine eher untergeordnete Rolle - vorausgesetzt, es wird überhaupt eine begleitende Fortbildung angeboten. Anders verhält es sich bei den Bibliothekartagen. Für den Bibliothekartag als typische Veranstaltung des Bildungsbereiches steht der Kongressanteil im Zentrum. Ein Blick auf die Erwartungen der Teilnehmerinnen und Teilnehmer kann, wie Tab. 2 veranschaulicht, näheren Aufschluss darüber geben, worin diese den Nutzen des Bibliothekartags für sich persönlich sehen.

5 Der wirtschaftliche Gesamtbetrieb ist bereits seit 2008 an professionelle Kongressagenturen (PCO) ausgelagert. 
Hierbei fällt auf, dass die Hauptinteressen am Bibliothekartag nur geringen Wandlungen unterworfen sind. Wenn man z. B. den Erwartungshorizont der Teilnehmerinnen und Teilnehmer der Bibliothekartage 2011 und 2015 vergleicht, zeigen sich durchgängige Trends.

Tab. 2: Hauptinteressen der Teilnehmerinnen und Teilnehmer.

\begin{tabular}{lll}
\hline $\begin{array}{l}\text { Hauptinteresse am Bibliothekartag } \\
\text { (Mehrfachnennung möglich) }\end{array}$ & Berlin 2011 & Nürnberg 2015 \\
\hline Beruflicher Austausch & $66,60 \%$ & $67,80 \%$ \\
\hline Themen des wissenschaftlichen Bibliothekswesens & $65,50 \%$ & $66,60 \%$ \\
\hline Themen des Bibliotheks- und Informationswesens generell & $60,80 \%$ & $50,70 \%$ \\
\hline Angebote der Firmenaussteller & $41,30 \%$ & $44,50 \%$ \\
\hline Themen des öffentlichen Bibliothekswesens & $22,00 \%$ & $22,80 \%$ \\
\hline Bibliotheken vor Ort in der Region & $16,60 \%$ & $11,00 \%$ \\
\hline Weitere Themen & $9,80 \%$ & $10,00 \%$ \\
\hline
\end{tabular}

Eines der Hauptinteressen von zwei Dritteln der Teilnehmerinnen und Teilnehmer am Bibliothekartag gilt dem beruflichen Austausch. Gleich danach folgen Themen des wissenschaftlichen Bibliothekswesens und des Bibliothekswesens generell. Für nahezu die Hälfte der Teilnehmerinnen und Teilnehmer stellt die Firmenausstellung eines ihrer Hauptinteressen dar, wobei diese Gruppe 2015 gegenüber 2011 gewachsen ist. ${ }^{6}$ Die Wirkung und der Nutzen des Bibliothekartags - bestimmt durch die Erwartungen der Besucherinnen und Besucher - bemessen sich demzufolge vornehmlich an den Möglichkeiten zum beruflichen Austausch. Thematische Interessen richten sich vor allem auf das wissenschaftliche Bibliothekswesen und spartenübergreifende Inhalte, wobei das Interesse an allgemeinen Themen des Bibliothekswesens zurückzugehen scheint. Die Firmenausstellung steht demgegenüber etwas zurück, ist aber ein bedeutender Teil des Kongressprogramms. Wert und Nutzen des Bibliothekartags dürften sich - neben der Qualität des Fortbildungsprogramms und der Firmenausstellung - also sehr stark daran orientieren, ob man dort fachkundige Kolleginnen und Kollegen antrifft.

Die Wirkung des Kongresses als Fortbildungsveranstaltung wird auch von den Beitragsarten stark beeinflusst. So wurde von den Teilnehmerinnen und

6 Vgl. Rosenberger, Burkard u. Wilfried Sühl-Strohmenger: 100. Deutscher Bibliothekartag Berlin 2011. Feedback der Teilnehmer(innen). https://www.vdb-online.org/wordpress/wp-content/uploads/2011/12/Bibliothekartag_Berlin2011_Teilnehmerfeedback_Auswertung_Okt2011. pdf; Rosenberger, Burkard u. Wilfried Sühl-Strohmenger: 104. Deutscher Bibliothekartag Nürnberg 2015. Teilnehmerfeedback/Entwurf der Auswertung (nicht veröffentlicht). 
Teilnehmern zuletzt immer wieder hervorgehoben, dass Workshops eben Workshop-Charakter haben und nicht einfach nur eine Vortragsfolge darstellen sollten. Die Möglichkeit, durch eigenes Mitarbeiten neues Methodenwissen zu erwerben, wird seit 2017 durch die so genannten Hands-on Labs und Project Labs verstärkt geboten. Aufgrund der zeitlichen Erstreckung des Bibliothekartags kam ein Vorschalten dieser Angebote vor den eigentlichen Kongress nicht infrage, weil nicht alle Teilnehmerinnen und Teilnehmer über die volle Laufzeit anwesend sind. Entsprechend finden die Labs während der gesamten Kongressdauer statt, erfordern zugleich aber umfangreiche Vorbereitungen und hohen finanziellen Aufwand, weil die jeweils passende Arbeitsumgebung bereitgestellt werden muss.

Die Wirkung des Bibliothekartags wird noch einmal dadurch erhöht, dass eine Verknüpfung von Kongress, kurzfristiger Internetberichterstattung und nachhaltigen Fachpublikationen stattfindet. Bei der kurzfristigen Berichterstattung kommt unterschiedlichen Communityblogs ${ }^{7}$ sowie den Kongressnews ${ }^{8}$ der Zeitschrift b.i.t.online die Hauptrolle zu. Ergänzend werden die Vortragsfolien der Teilnehmerinnen und Teilnehmer noch während des Kongresses auf dem OPUS-Server des BIB zur Verfügung gestellt. ${ }^{9}$ Der eigentliche Tagungsband der Bibliothekartage erscheint seit Gründung der Open-Access-Zeitschrift $o$-bib durch den VDB regelmäßig als Heft 4 der Zeitschrift. Dafür wird ein offener Call gestartet und alle Beiträge durchlaufen anschließend ein Peer-Review-Verfahren. Bei der Kommunikation des Bibliothekartags wirken also ganz unterschiedliche Player und Medien zusammen, und dies trägt zur Nachhaltigkeit des Kongresses wesentlich bei.

\section{Rolle der Verbände}

Der Bibliothekartag ist traditionell die wichtigste Veranstaltung der bibliothekarischen Personalverbände. Nicht nur geht das Angebot der beiden Verbände

\footnotetext{
7 Besonders erwähnt seien hier der Studierendenblog Bibliotheksnews (https://bibliotheksnews.com/) der HAW Hamburg in Kooperation mit b.i.t.online sowie der Blog Bibliothekarisch von Dörte Böhner.

8 http://www.b-i-t-online.de/daten/kn.php.

9 Dazu dient der vom BIB betriebene OPUS-Server: https://opus4.kobv.de/opus4-bib-info/solrsearch/browse.
} 
über das reine Networking auf einem jährlichen Kongress weit hinaus, auch kann umgekehrt die Bedeutung des Bibliothekartags für die Personalverbände wohl gar nicht hoch genug eingeschätzt werden. So erreichen BIB und VDB mit diesem Angebot regelmäßig einen großen Teil ihrer Mitglieder: jedes Mal bis zu $16 \%$ bzw. $30 \%$.

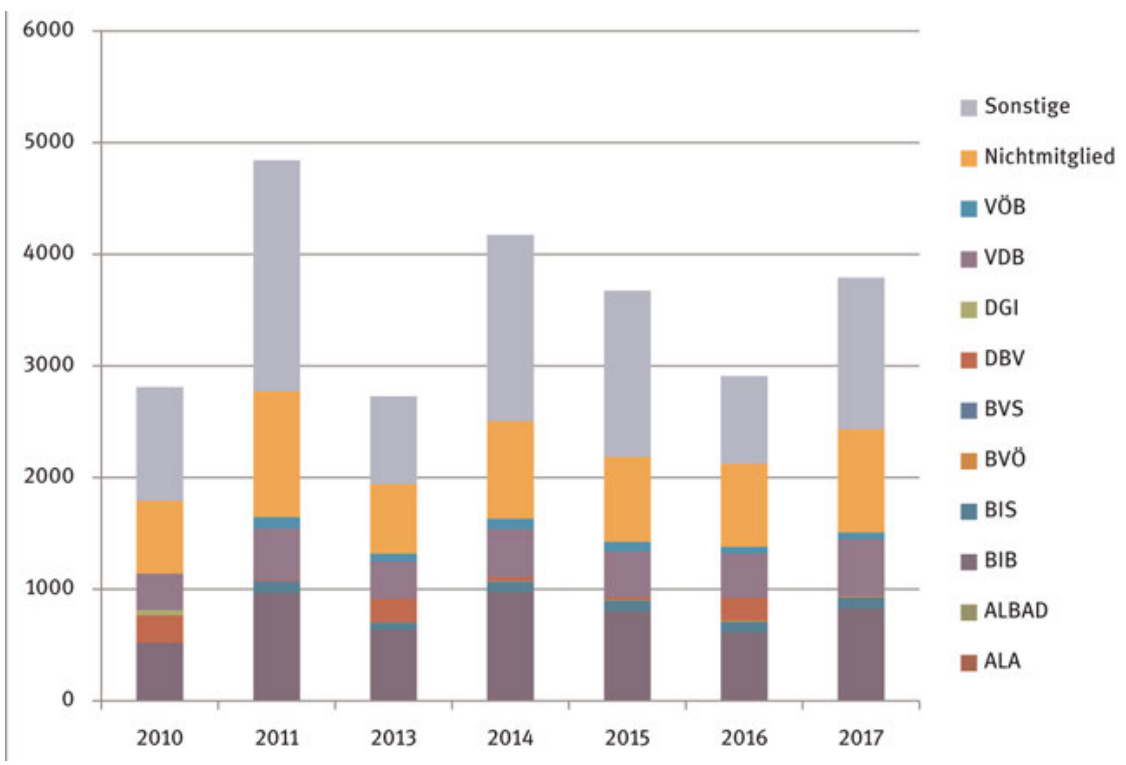

Abb. 4: Teilnehmerinnen und Teilnehmer an Bibliothekartagen und Bibliothekskongressen nach Verbandszugehörigkeit. ${ }^{10}$

Weiterhin erwachsen aus der Arbeit der Kommissionen und anderen Fokusgruppen der beiden veranstaltenden Verbände viele Programminhalte und wirken in die Verbände zurück. Nicht wenige neue Angebote entstehen aus dem Bibliothekartag heraus, insbesondere neue Fortbildungsangebote, die von den Denkimpulsen der Kongresse leben. Schließlich dient der Bibliothekartag nicht nur der Verbandsarbeit von BIB und VDB, sondern auch einer Reihe anderer Organisationen, Verbände und Arbeitsgruppen als Plattform für ihre inhaltliche Arbeit ebenso wie für sonstige Verbandstermine. Hier ist von einem wechsel-

10 Die sonstigen Teilnehmerinnen und Teilnehmer setzen sich aus Referenten, Ausstellern, ermäßigten Teilnehmern sowie dem Organisationsteam zusammen; die Verbandszugehörigkeit wird in diesen Fällen nicht erfasst. 
seitigen Geben und Nehmen bei der Infrastruktur und den Programminhalten auszugehen, welches die Zusammenarbeit für beide Seiten attraktiv macht. Zugleich ist der Bibliothekartag offen und flexibel genug für ad-hoc-Arbeitsgruppen und weniger festgefügte Communitys, die das Programm zunehmend prägen. Die Rolle der veranstaltenden Verbände besteht darin, die unterschiedlichen Anliegen zu moderieren und aufzunehmen und nicht zuletzt das wirtschaftliche Risiko für das Gesamtunternehmen zu tragen.

\section{Bibliothekartag 4.0}

Um im Wettbewerb um die Informationssuchenden auch in Zukunft zu bestehen, wird sich der Bibliothekartag weiter verändern und den Fortbildungsgewohnheiten anpassen müssen. Dabei dürften der Erhalt und weitere Ausbau des Kongresscharakters und die Kombination mit einer großen Firmenmesse die Essentials darstellen. Thematisch sollte der Schwerpunkt auf den Wachstumsbereichen liegen. Gerade im Bereich der Technologieunterstützung besteht ein hoher Bedarf beim Wissenstransfer. Hier müssen sich aber auch die Vermittlungsformen ändern - Stichwort virtuelle Tagungen, Veranstaltungen 4.0 und hybride Events. Der Bibliothekartag der Zukunft wird - genau wie andere Kongresse und Messen - immer stärker Teil eines Netzwerks und weniger abgeschlossener Event sein, wenn er erfolgreich sein soll. Zunehmende individuelle oder gruppenspezifische Ansprüche bei Teilnehmerinnen und Teilnehmern und Veranstaltern, vermehrte Interaktivität in Verbindung mit neuen Technologien und der Bedarf nach stärkerer Nachhaltigkeit bei Wissensaustausch und Fortbildungseffekt werden diese Entwicklung charakterisieren. Das Bedürfnis nach paralleler Teilnahme mittels Videostreaming oder Social-Media-Begleitung wächst. Von den Veranstaltern erfordert dies ein gewisses „Loslassen“, könnten sich doch Inhalte verselbständigen oder der wirtschaftliche Betrieb leiden. Mit durchinszenierten Ereignissen das Interesse an einer Vor-Ort-Partizipation zu bedienen, um damit ein Gegenmittel zur Digitalisierung des Kongresses zu schaffen, dürfte jedoch der falsche Weg sein. Im Gegenteil ist es Teil der Erfolgsgeschichte des Bibliothekartags, dass Inhalte und Ergebnisse offen für die Community bereitgestellt werden. Der Bibliothekartag ist ein Inkubator für Innovationen und für den Kulturwandel im Bibliothekswesen. Mit zunehmender virtueller Vernetzung der Zielgruppe tritt auch auf dem physischen Event der persönliche Austausch gegenüber den eigentlichen Präsentationen noch stärker in den Vordergrund. Elemente des gemeinsamen Tuns und Problemlösens wer- 
den wichtiger. So kann die Nachhaltigkeit des Kongresses gestärkt werden. Je komplexer und virtueller der Bibliothekartag als Fortbildungsgeschehen wird, umso wichtiger könnte schließlich dessen Charakter als Dachmarke werden. 